Check for updates

Cite this: RSC Adv., 2018, 8, 29141

Received 4th July 2018

Accepted 7th August 2018

DOI: $10.1039 / c 8 r a 05698 f$

rsc.li/rsc-advances

\title{
Electrical properties of O-self-doped boron-nitride nanotubes and the piezoelectric effects of their freestanding network film $\uparrow$
}

\begin{abstract}
Chuncheng Ban, (D) ${ }^{a}$ Ling Li ${ }^{\star a b c}$ and Liuxiao Wei ${ }^{a b c}$
Boron-nitride nanotubes (BNNTs) are a common one-dimensional (1D) nanostructure that possess piezoelectric potential due to ion-covalent boron-nitride (BN) bonding. Harnessing the advantages offered by high-stability BN structures, these materials have been used for various new applications such as nanogenerators, nanotransistors, and nano-artificial eardrums. In this paper, we used nano-iron oxide red as a catalyst and boron powder in an aqueous dispersion as the boron source to synthesize highpurity O-self-doped BNNTs and film. We investigated the electrical properties of O-self-doped BNNTs and the piezoelectricity of freestanding BNNT film and demonstrated that the electrical properties of O-self-doped BNNTs improved dramatically compared to those of non-doped BNNTs. We also analyzed the band gaps and density of states (DOS) of the O-self-doped BNNTs with the Spanish Initiative for Electronic Simulation with Thousands of Atoms (SIESTA) code to explain the improvement. In addition, we revealed the piezoelectric voltage coefficient $g_{31}$ of $O$-self-doped BNNTs $\left(0.28 \mathrm{~V} \mathrm{~m} \mathrm{~N} \mathrm{~m}^{-1}\right)$ network films, which can guide future applications for vibration nanosensors and transducers under extreme conditions.
\end{abstract}

\section{Introduction}

1D nanostructures can be made of many types of NT materials, such as carbon NTs (CNTs), BNNTs, zinc oxide NTs, and titanium dioxide NTs. Wide band gap (5.6 eV) BNNTs have the same crystalline structure as carbon NTs. ${ }^{1,2}$ Compared to other 1D nanostructures, BNNTs exhibit high oxidation-thermal stability and chemical inertness. ${ }^{3}$ However, limited synthetic methods have encouraged some researchers to focus on the composite films inside BNNTs. These studies have revealed some promising properties, including linear piezoelectric and nonlinear electroactive strain, thermal, electrical, and mechanical properties of composite films. ${ }^{4-8}$ Furthermore, the piezoelectric potential distribution under an applied stress has been attributed to the noncentral symmetry of BNNTs. ${ }^{9}$ Currently, these unique properties of BNNTs are utilized in many applications, such as nanotransistors, nanosensors, nanogenerators and nanopiezoelectricity. ${ }^{10,11}$ ${ }^{a}$ MEMS Center, Harbin Institute of Technology, Harbin, 150001, China. E-mail:
linglimems@hit.edu.cn
${ }^{b}$ State Key Laboratory of Urban Water Resource \& Environment (Harbin Institute of
Technology), Harbin 150001, China
${ }^{c}$ Key Laboratory of Micro-systems and Micro-Structures Manufacturing, Ministry of
Education, Harbin 150001, China

$\dagger$ Electronic supplementary information (ESI) available. See DOI: 10.1039/c8ra05698f
Although BNNTs possess many advantages beneficial for practical applications, some major obstacles prevent their piezoelectric application, including high impedance and purity and yield. Many researchers are searching for elements and methods that are more efficient for BNNT doping to decrease resistance, and many studies have focused on theoretical simulations based on the Kohn-Sham equations. For instance, some studies investigated Au-doped BNNTs, V-doped BNNTs and C-doped BNNTs. ${ }^{12-14}$ However, doping with larger atomic number elements has been challenging because these elements only adsorb on the NT surface. In addition, the purity and yield of C-doped BNNTs is limited. Motivated by these issues, $\mathrm{O}$ element doped BNNTs seems to be a better choice. Moreover, recently reports reveals $\mathrm{O}$ doped $\mathrm{BN}$ material process an excellent ferromagnetic properties at room temperature, and other attracting properties. ${ }^{\mathbf{1 5 , 1 6}}$ So that, this properties suggest that $\mathrm{O}$ doped BNNT is likely to a very promising piezoelectric nanomaterial for a widely application.

In this paper, a high purity, high yield and stable O-selfdoped BNNTs were synthesized by a nano-ferric oxidecatalyzed boron annealing method in this article. These BNNTs were assembled into a freestanding film via vacuum filtration of a BNNT suspension, which is similar to the buckypaper of CNTs. ${ }^{17}$ We investigated the electrical properties of the O-self-doped BNNTs and their network film using thermionic-field emission theory. With further research, we determined that the band gap of the O-self-doped BNNTs decreased upon the introduction of a donor level in the BNNTs, 
which agreed with some calculations based on the density functional theory (DFT). ${ }^{18}$ In addition, we investigated for the first time the piezoelectric coefficient of the randomly oriented pure O-self-doped BNNT network film without any polymer. Using these factors and the relative dielectric constant, piezoelectric strain coefficient can be estimated. ${ }^{19}$

\section{Experimental}

\section{Synthetic procedures}

The O-doped BNNTs were synthetized using nano-iron oxide red as a catalyst at high temperature, and a schematic diagram of BNNT preparation system is shown. First, amorphous boron powder (ALDRICH, $\geq 95 \%$ amorphous boron power) and nanosized $\mathrm{Fe}_{2} \mathrm{O}_{3}$ powder (Aladdin, $30 \mathrm{~nm} \mathrm{99.5 \%} \mathrm{Fe}_{2} \mathrm{O}_{3}$ ) with a high surface area were mixed. Next, $0.2 \mathrm{~g}$ of the mixed powder was placed into vials filled with ammonia water in a glove box under $\mathrm{N}_{2}$ atmosphere to prepare the raw material for the synthesis process. After ultrasonicating the raw material for $10 \mathrm{~min}$, the raw ink material was coated on a cleaned stainless-steel substrate. Finally, the stainless-steel substrate was placed into a tube furnace at $1150{ }^{\circ} \mathrm{C}$, and the furnace was filled with $\mathrm{N}_{2}+$ $15 \% \mathrm{H}_{2}$ to synthesize BNNTs.

\section{BNNT network film preparation method}

The BNNTs were peeled from the stainless-steel substrate and still contained some impurities and the catalyst. The purity of NTs in the suspension greatly affected the quality of the BNNT network film and was key to successful preparation of the film. To increase the purity of the BNNTs, a wash and oxidation method with nitric acid at a high temperature was performed. We then used ultrasonic dispersion to obtain a uniformly dispersed BNNT suspension solution in ethyl alcohol with a concentration of $2.5 \mathrm{mg} \mathrm{ml} \mathrm{m}^{-1}$. The BNNT network film was fabricated using $20 \mathrm{ml}$ of the suspension with microfiltration membrane paper. The polytetrafluoroethylene microfiltration membrane paper (PTFE) had a characteristic $0.22 \mu \mathrm{m}$ pore size and was attached to a $13 \mathrm{~mm}$ Luer syringe filter. As the solvent evaporated under a low vacuum at room temperature, the local concentration of BNNTs near the membrane rapidly increased, and BNNTs were deposited on the microfiltration paper to form a paper-like network film as shown in Fig. S2 and S3. $\dagger$

\section{First principles calculation}

Calculations were performed using the SIESTA code, which is based on $\mathrm{DFT}^{29}$ All the calculations were based on a first principles pseudopotential method within the GGA, and the PBE form was adopted as the exchange-correlation functional. ${ }^{\mathbf{4 1 4 2}}$ Norm-conserving pseudopotentials for all the atoms were generated using the standard conserved KleinmanBylander pseudopotential for the completely nonlocal form. A cut-off of 400 Ry for grid integration and a double-zeta plus polarization (DZP) basis set were utilized for these calculations. ${ }^{28}$ Monkhorst-Pack $k$-points were employed as sets of special points in the Brillouin zone considering only the axial direction of the NT for a 1D material. The structural optimizations were performed using a conjugated gradient (CG) procedure until the residual forces were smaller than $0.05 \mathrm{eV}$ $\AA^{-1}$. Based on this parameter, we calculated the band gap and DOS of the O-self-doped BNNTs.

\section{Testing and characterization equipment}

The morphologies and chemical compositions of the products were investigated using SEM (VRGA 3 SBH, TESCAN, Czech Republic) equipped with an EDX spectroscopy system (NORAN System 7, Thermo Scientific, USA). XPS (Thermo Fisher Scientific, ESCALAB 250Xi, USA) was used to analyze the bands and elements in the NTs. A JEOL-2100 transmission electron microscope (JEOL Ltd., Akishima, and Tokyo, Japan) was used to investigate the structure of the NTs. $I-V$ curves were measured using a precision semiconductor parameter analyzer (Keysight technologies Ltd., B1500a, USA) with a four-tungstenprobe shielding box. A magnetron sputtering coating machine (JZCK-400, Liaoning Juzhi Technology Company Ltd., China) sputtered the electrode on the surface of the BNNTs. The strain or stress on the BNNT network film was supplied by an accurate mass counterweight on the surface of the film using gravity.

\section{Results and discussion}

Our studies of O-self-doped BNNTs included two parts: the novel preparation of freestanding NT film and an investigation of the electrical properties and piezoelectric characteristic of outside pressure on the network film. We focused on the effects of $\mathrm{O}$ on the conductive capacity and the piezoelectricity of BNNT film. Therefore, there were three aspects that must be addressed. First, the purity and yield of the nano- $\mathrm{Fe}_{2} \mathrm{O}_{3}$ catalyzed annealing method was described. Next, an analysis of how BNNT conductivity was affected by $\mathrm{O}$ was performed, and finally, the piezoelectricity of O-self-doped BNNT film was characterized. In this paper, the nano- $\mathrm{Fe}_{2} \mathrm{O}_{3}$ catalysed annealing method was used to efficiently synthesize NTs, and the detailed experiment process is described in the following

a)

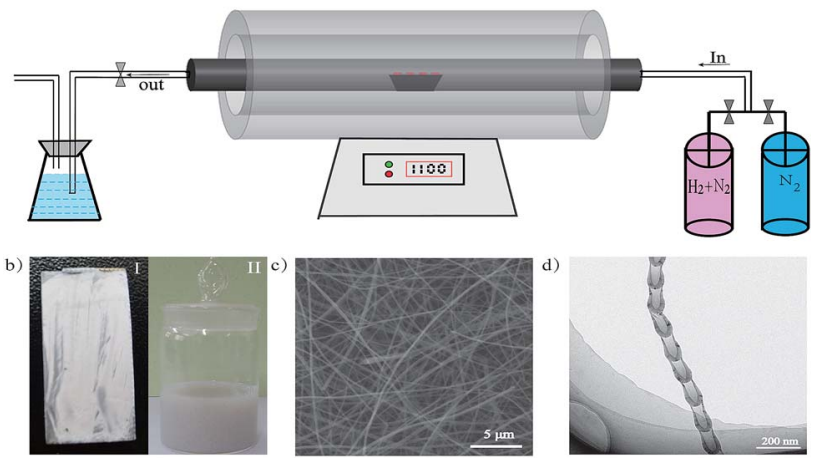

Fig. 1 (a) Schematic diagram of the BNNT preparation system. (b) I shows a physical map of O-self-doped BNNTs on the substrate, II shows a high-purity O-self-doped BNNT suspension. (c) SEM image of the O-self-doped BNNTs (10 kV for SEM). (d) TEM images of the Oself-doped BNNTs (200 kV for TEM). 
section, the diagrammatic sketch of preparation equipment are shown in Fig. 1a. White, O-self-doped BNNTs on the substrate with a macrograph map are shown in Fig. 1b-I, and NTs dispersed as a liquid in alcohol are shown in Fig. 1b-II. Scanning electron microscopy (SEM) images showed that the NTs were more than $30 \mu \mathrm{m}$ long, and the diameters ranged from $50 \mathrm{~nm}$ to $120 \mathrm{~nm}$ as shown in Fig. 1c. TEM images indicate that the NTs have a bamboo shape with a diameter of approximately $60 \mathrm{~nm}$, as shown in Fig. 1d. These morphological features are a compelling demonstration of the high purity and yield of NTs formed with the nano- $\mathrm{Fe}_{2} \mathrm{O}_{3}$ catalyzed annealing method. Here, we must note previous methods for preparing $\mathrm{O}$ none-doped BNNTs based on the boron ball-milling-annealing method, which exhibit a similar bamboo BNNT growth mechanism. ${ }^{20}$

A schematic diagram of the simplified preparation model in section view is shown in Fig. 2. The simplified growth mechanism of NTs involves five steps. The first step is to completely mix boron powders with the $\mathrm{Fe}_{2} \mathrm{O}_{3}$ nanoparticles. Next, the boron powders provide $\mathrm{B}$ raw materials for NTs and adsorb on the surface nano- $\mathrm{Fe}_{2} \mathrm{O}_{3}$ particles before annealing. The third step requires the following chemical reactions to occur on the surface of $\mathrm{Fe}_{2} \mathrm{O}_{3}$ nanoparticles during annealing:

$$
\mathrm{H}_{2(\mathrm{~g})}+\mathrm{N}_{2(\mathrm{~g})}+n \mathrm{~B}_{(\mathrm{s})} \stackrel{\mathrm{Fe}_{2} \mathrm{O}_{3}}{\Rightarrow} \mathrm{BN}_{(\mathrm{s})}
$$

In these reactions, $\mathrm{H}_{2}$ is the activator, $\mathrm{Fe}_{2} \mathrm{O}_{3}$ is the catalyst, and $\mathrm{BN}$ localizes on the surface of the $\mathrm{Fe}_{2} \mathrm{O}_{3}$ nanoparticles. The next step is induced by the surface diffusion motion of $\mathrm{BN}$ on $\mathrm{Fe}_{2} \mathrm{O}_{3}$ nanoparticles to generate a full-closed $\mathrm{BN}$ shell layer on the surface. ${ }^{21}$ The final step uses capillarity effects to form the NTs at high temperature. ${ }^{22}$ During the growth process, we noted that the size of the catalyst nanoparticle and the annealing temperature substantially affected BNNT yield. ${ }^{23-25}$ In addition, during the fourth and fifth steps, some O elements were introduced into the BNNT crystal lattice, suggesting that the $\mathrm{O}$ element originates from $\mathrm{Fe}_{2} \mathrm{O}_{3}$ at the high temperature and randomly substitutes for $\mathrm{B}$ or $\mathrm{N}$ atoms during the annealing process in NTs. ${ }^{26}$ To analyze the role of $\mathrm{O}$, X-ray photoelectron spectroscopy (XPS) was used to analyze the elements in the NTs, as shown in the Fig. 3a; the XPS results showed that $\mathrm{O}$ was

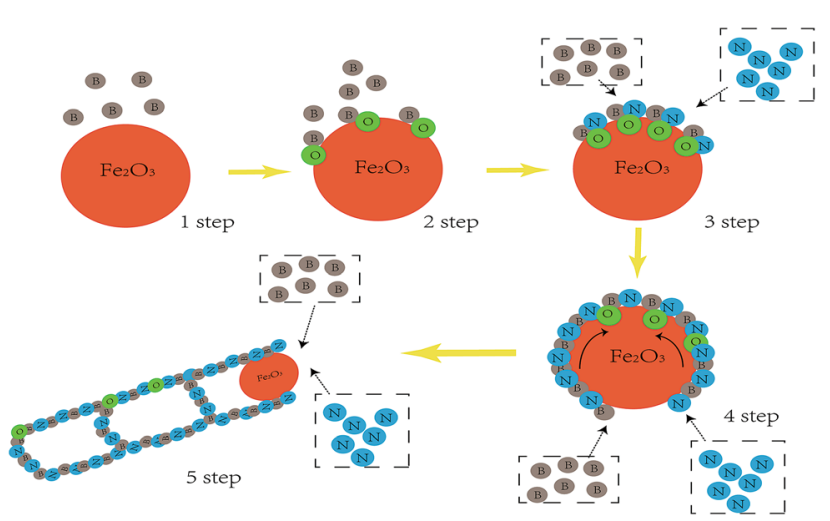

Fig. 2 Schematic diagram of the growth mechanism of the NTs in a section view.
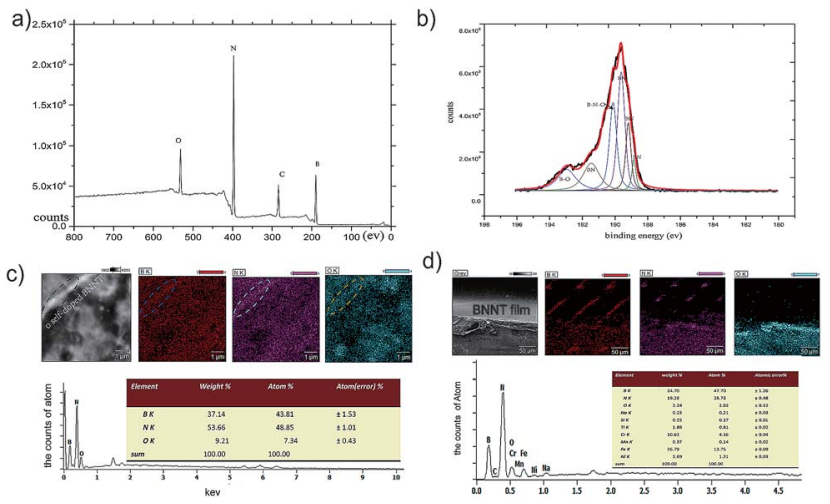

Fig. 3 (a) The elementary composition of O-self-doped BNNTs film. (b) Survey images and a B 1s scan with the XPS-peak-differentiationimitating analysis images of the O-self-doped BNNTs film. (c) EDX results and the elemental ratios of the O-self-doped BNNTs film, and the mapping analyse of the single NTs. (d) EDX results and element ratios of the pure BNNTs film and the mapping analyse of the film along the section orientation of substrate (NTs from the ball milling methods).

present in the BNNTs. The XPS results were coincident. We fitted the B 1s peak with a scatter peak to determine the different bonds (the carbon peak at $284 \mathrm{eV}$ comes from the environment, and rectification of the peak is required), which is shown in Fig. 3b. Moreover, the energy dispersive X-ray (EDX) results showed the doping atomic ratio of $\mathrm{O}$ in the $\mathrm{O}$-self-doped NTs reached $7.34 \%$ (Fig. 3c) and the none-doped NTs reached $2.93 \%$ (Fig. 3d). Fig. 3c shows the EDX mapping analyse of single NT as a clear proofs to indicate the reality of oxygen doping. Combine with the results of the mapping analyse of none-doped BNNT, we suggest that the little O element was possible from the impurities of substrate. Based on the NIST Xray Photoelectron Spectroscopy Database, the $\mathrm{B}-\mathrm{O}$ and $\mathrm{B}-\mathrm{N}-\mathrm{O}$ bonds were included in the NTs, suggesting that $\mathrm{O}$ substitutes for $\mathrm{B}$ or $\mathrm{N}$ atoms randomly. ${ }^{27}$ Based on the results above, we confirm that the nano- $\mathrm{Fe}_{2} \mathrm{O}_{3}$ catalyzed annealing method efficiently synthesizes O-self-doped BNNTs with high purity.

To understand the improved conductivity of NTs after O doping, we first generated a physical model based on DFT to explain this effect. ${ }^{28}$ The $(6,0)$ and $(8,8)$ BNNT xyz coordinate files were built via the Virtual Nanolab as a precursor for simulation as shown in Fig. S1. $\dagger^{29}$ We used a generalized gradient approximation (GGA) Perde-Burke-Ernzerhof (PBE) model with an exchange-correlation energy and a SIESTA code based on the Linux system to obtain the band gap and DOS of the O-self-doped $(6,0$ and 8, 8) BNNTs to compare with those of the non-doped BNNTs. We simulated substrates with $\mathrm{O}$ substituted for $\mathrm{N}$ ( $\mathrm{O}_{\mathrm{N}}$-doped) and $\mathrm{B}\left(\mathrm{O}_{\mathrm{B}}\right.$-doped) as shown in Fig. S4. $\dagger$ After completing the calculations, the band gap of the doping lattice with oxygen decreased in the $(8,8)$ armchair and $(6,0)$ zigzag BNNTs as shown in Fig. S5. $\uparrow$ A narrower band led to higher conductivity in the NT, which generated the observed increased current. All of the DOS and energy band structures from impurities were observed at the expected donor energy levels (refer to ESI† for details). 
Next, we studied the electrical characteristics of O-self-doped BNNTs by high-precision equipment. We obtained a Ni/Au electrode with lift-off photolithography (Fig. 3-I) after the magnetron sputtering process in the prevacuum chamber (Fig. 3-II). As seen in Fig. 3a-III, the blue curve is the current of the O-self-doped NTs, which reached $4 \mu \mathrm{A}$ at their highest and showed a test voltage range from $-5 \mathrm{~V}$ to $+5 \mathrm{~V}$. Our results show that the electrical properties of the O-self-doped BNNTs improved substantially compared to the non-doped BNNTs (the red curves). Pure NTs were synthesized using our previous ball milling-annealing methods with a long milling time, as shown in Fig. $3 \mathrm{~d} .{ }^{20}$ To decrease the contact barrier, the testing electrode system required a crystallization process by annealing at $525^{\circ} \mathrm{C}$ in air. ${ }^{30-32}$ Here, it seems that the Stark effect, the electron spin effect, or other quantum effects might account for the decreased barrier, but additional studies are required. ${ }^{33,34}$ According to the thermionic-field emission theory, the first order differential $I-V$ curve is associated with NT resistance: $R \approx \mathrm{d} V / \mathrm{d} I{ }^{35}$ According to this first order differential equation, resistance has the value $R_{\text {non-doped }} \approx 1.3 \times 10^{9} \Omega$ and $R_{\text {O-self-doped }} \approx 0.85 \times 10^{6} \Omega$. In general, conductance is the reciprocal of resistance, indicating that the electrical conductivity of O-doped BNNTs was much higher than that of non-doped BNNTs, and the results these tests are in agreement with the theory.

In view of the vast improvement in electrical properties, we characterized the piezoelectric properties of O-self-doped BNNT network film and calculated the piezoelectric voltage coefficient $g_{31}$ based on piezoelectric constitutive equations (PCE) and apposite boundary conditions to explore potential in piezoelectric sensors. ${ }^{36,37}$ The thermal annealing condition was applied on the electrode to create the contact well, which is similar to a single NT. The network film was from the vacuum filtration of an ethanol suspension. Fig. $4 \mathrm{~b}$ shows the $I-V$ curves of NT network films under different pressures and that the
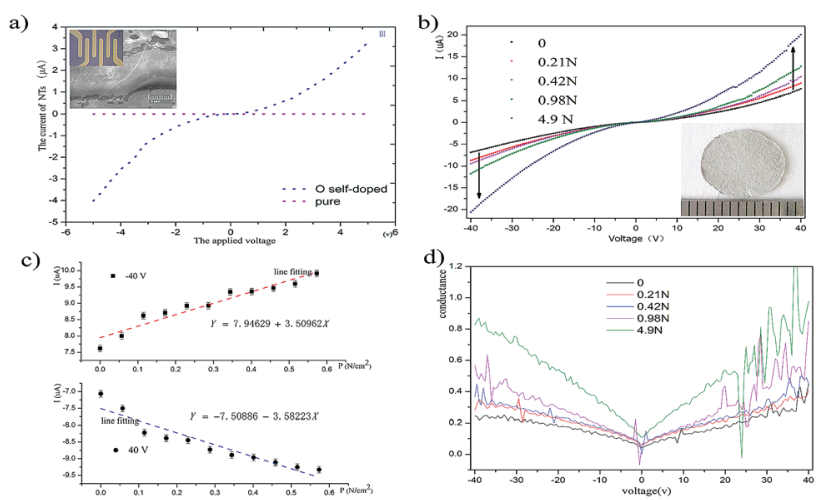

Fig. 4 (a) I shows the electrode structure manufactured by the lift-off process, II shows the SEM image of the O-self-doped single NT contacting the electrode, and III shows the I-V curves of single O-selfdoped (blue) and pure (purple) BNNTs in a voltage range of -5 to $5 \mathrm{~V}$. (b) Piezoelectric properties of the BNNT network film under different forces and a physical map of the O-self-doped BNNT network film. (c) Images show the change in current with pressure at $-40 \mathrm{~V}$ and $40 \mathrm{~V}$, respectively. (d) The conductance $v s$. the voltage curves at the different pressures. current increases with increasing pressure. Obviously, the conductance of network film increases with pressure due to the piezoelectric effect, as shown in Fig. 4d. Fig. 4c shows the curves for film current I $v s$. stress $\mathrm{S}_{3}$ at $40 \mathrm{~V}$ and $-40 \mathrm{~V}$. The red line, $K_{1}$, and the blue line, $K_{2}$, in Fig. $4 \mathrm{c}$ are represented by the linear fitting slope at two different voltages; here, the slope $K=\Delta I / T(T$ for the stress) is relevant to the piezoelectric coefficient. The network film was sandwiched between the slide and the coverslip to maintain zero strain and electrically insulate the surroundings. Therefore, this experiment fitted to the third boundary condition of the piezoelectric equation.

The piezoelectric properties of the BNNTs come from their non-centrosymmetric hexagonal BN structure, and polarization resulting in piezoelectric properties can be strain controlled or stress controlled. ${ }^{38,39}$ It is known that the piezoelectric coefficient $d_{31}$ is significantly correlated with piezoelectric effect performance. In addition, the piezoelectric coefficient indicates the polarization of a piezoelectric material upon application of a stress: $d_{31}=D_{3} / T_{1}$, in which $D$ is the electric displacement, and $T$ is the stress. For the linear electrical behavior of the material, $D_{3}=\varepsilon E_{3}$, in which $\varepsilon$ is permittivity (free-body dielectric constant), and $E$ is the electric field strength. Here, electric field $E$ is related to resistivity and current density $J$, and current density $J$ is related to current $I$. Therefore, the piezoelectric coefficient $d_{31}$ was calculated using the follow equation:

$$
d_{31} \approx \frac{\varepsilon_{\mathrm{BNNT}} \Delta I R_{\mathrm{film}}}{T_{3} d_{1}}=\varepsilon_{\mathrm{BNNT}} K \frac{R_{\mathrm{film}}}{d_{1}}
$$

In which $\Delta I$ is the change of current, $T_{3}$ is the load stress applied on the surface of the NTs film, and $d_{1}$ is the electrode gap $1.5 \mathrm{~mm}$. In addition, $d_{31}=g_{31} / \varepsilon_{\mathrm{BNNT}}$, with $\varepsilon_{\mathrm{BNNT}}$ approximately equal to 5.9 times vacuum permittivity. According to eqn (1) and the $I-V$ curves of the film without stress, the resistance $R_{\text {film }} \approx 2.5 \mathrm{M} \Omega$. Therefore, the piezoelectric coefficient $d_{31} \approx$ $31.186 \mathrm{pC} \mathrm{N}^{-1}$, and $g_{31} \approx-0.597 \mathrm{~V} \mathrm{~m} \mathrm{~N}^{-1}$. The values for the $d_{31}$ coefficient are similar to other reports, and the $g_{31}$ coefficient of the BNNT film is 2 times higher than the PVDF film (Max $g_{31}=$ $\left.0.28 \mathrm{~V} \mathrm{~m} \mathrm{~N}^{-1}\right) .{ }^{40}$ Significantly, these sample methods might resolve the primary challenge in regard to measuring the piezoelectric constant.

\section{Conclusions}

In this article, high-quality O-doped BNNTs were fabricated using a nanocatalyst-assisted annealing method. This method makes the study of BN nano material fast. The O self-doped BNNT network films were prepared using a microporous filtration method, and the electrical properties of the $\mathrm{O}$ selfdoped single BNNT and the network film were investigated using an $I-V$ test and calculation. The conductivity of O-doped BNNTs was much higher than that of non-doped BNNTs, indicating that $\mathrm{O}$ markedly improves the electrical properties of BNNTs. We illustrated that the change in the energy band structure and DOS of O-self-doped BNNTs may cause increased electrical properties based on a first principle. The assessment of the BNNT film also clearly demonstrated its piezoelectric 
characteristics. This higher conductivity of piezoelectric $\mathrm{O}$ doped BN 1D nano material as a widely attraction material applied in the piezoelectric nano-devices, 1D electric nanodevices, and nano generator. Moreover, we calculated the piezoelectric constant $d_{31}$ and $g_{31}$ using a sample method. Herein, the $g_{31}$ coefficient described the change in the electric field when stress was changed. The results show that BNNT network film had a higher piezoelectric voltage coefficient, indicating its high sensitivity to a supersonic wave compared to PVDF film. These results can guide the development of nano transducers, nano medicine and nano wearable devices.

\section{Conflicts of interest}

There are no conflicts to declare.

\section{Acknowledgements}

This work is financially supported by the Joint Space Science and Technology Fund (No. 6141B06260302), the National Natural Science Funds of China (Grant No. 61404036), and the State Key Laboratory of Urban Water Resource and Environment (Harbin Institute of Technology) (No. 2016TS 06).

\section{Notes and references}

1 D. Golberg, Y. Bando, K. Kurashima and T. Sato, Synthesis and Characterization of Ropes Made of BN Multiwalled Nanotubes, Scr. Mater., 2001, 44, 1561-1565.

2 R. H. Baughman, C. Cui, A. A. Zakhidov, Z. Iqbal, J. N. Barisci, G. M. Spinks, G. G. Wallace, A. Mazzoldi, R. D. De and A. G. Rinzler, Carbon Nanotube Actuators, Science, 1999, 284, 1340.

3 Y. Chen, J. Zou, S. J. Campbell and G. L. Caer, Boron Nitride Nanotubes: Pronounced Resistance to Oxidation, Appl. Phys. Lett., 2004, 84, 2430-2432.

4 T. Terao, C. Zhi, Y. Bando, M. Mitome, C. Tang and D. Golberg, Alignment of Boron Nitride Nanotubes in Polymeric Composite Films for Thermal Conductivity Improvement, J. Phys. Chem. C, 2010, 114, 4340-4344.

5 K. S. Kim, M. B. Jakubinek, Y. Martinez-Rubi, B. Ashrafi, J. Guan, K. O'Neill, M. Plunkett, A. Hrdina, S. Lin and S. Denommee, Polymer Nanocomposites from FreeStanding, Macroscopic Boron Nitride Nanotube Assemblies, RSC Adv., 2015, 5, 41186-41192.

6 J. H. Kang, G. Sauti, C. Park, V. I. Yamakov, K. E. Wise, S. E. Lowther, C. C. Fay, S. A. Thiebeault and R. G. Bryant, Multifunctional Electroactive Nanocomposites Based on Piezoelectric Boron Nitride Nanotubes, ACS Nano, 2015, 9, 11942.

7 K. Khoo, M. S. C. Mazzoni and S. G. Louie, Electronic Properties of Boron Nitride Nanotubes in the Presence of a Transverse Electric Field, in APS Meeting, 2003.

8 Y. Gu, X. Wang, W. Gu, Y. Wu, T. Li and T. Zhang, Flexible Electronic Eardrum, Nano Res., 2017, 10, 2683-2691.
9 D. Golberg, Y. Bando, Y. Huang, T. Terao, M. Mitome, C. Tang and C. Zhi, Boron Nitride Nanotubes and Nanosheets, ACS Nano, 2010, 4, 2979-2993.

10 Z. L. Wang, Novel Nanostructures of ZnO for Nanoscale Photonics, Optoelectronics, Piezoelectricity, and Sensing, Appl. Phys. A: Mater. Sci. Process., 2007, 88, 7-15.

11 S. M. Nakhmanson, A. Calzolari, V. Meunier, J. Bernholc and M. B. Nardelli, Spontaneous Polarization and Piezoelectricity in Boron Nitride Nanotubes, Phys. Rev. B, 2003, 67, 102-105.

12 Y. J. Chen, L. Fu, Y. Chen, J. Zou, J. Li and W. H. Duan, Tunable Electric Conductivities of Au-Doped Boron Nitride Nanotubes, Nano, 2008, 2, 367-372.

13 K. L. Yao, Y. Min, S. C. Zhu, G. Y. Gao, Z. L. Liu and H. G. Cheng, First-Principles Study of Transport of $\mathrm{V}$ Doped Boron Nitride Nanotube, Phys. Lett. A, 2008, 372, 5609-5613.

14 J. X. Zhao and B. Q. Dai, DFT Studies of Electro-Conductivity of Carbon-Doped Boron Nitride Nanotube, Mater. Chem. Phys., 2004, 88, 244-249.

15 Q. Lu, Q. Zhao, T. Yang, C. Zhai, D. Wang and M. Zhang, Preparation of Boron Nitride Nanoparticles with Oxygen Doping and a Study of Their Room-Temperature Ferromagnetism, ACS Appl. Mater. Interfaces, 2018, 10, 12947-12953.

16 J. Sahu, K. Panda, B. Gupta, N. Kumar, P. A. Manojkumar and M. Kamruddin, Enhanced Tribo-Chemical Properties of Oxygen Functionalized Mechanically Exfoliated Hexagonal Boron Nitride Nanolubricant Additives, Mater. Chem. Phys., 2018, 207, 412-422.

17 M. Endo, H. Muramatsu, T. Hayashi, Y. A. Kim, M. Terrones and M. S. Dresselhaus, Nanotechnology: 'Buckypaper' from Coaxial Nanotubes, Nature, 2005, 433, 476.

18 G. Gou, B. Pan and L. Shi, The Nature of Radiative Transitions in O-Doped Boron Nitride Nanotubes, J. Am. Chem. Soc., 2009, 131, 4839.

19 J. F. S. Jr, P. J. Moses and S. Trolier-Mckinstry, The Wafer Flexure Technique for the Determination of the Transverse Piezoelectric Coefficient $\left(\mathrm{d}_{31}\right)$ of PZT Thin Films, Sens. Actuators, A, 1998, 71, 133-138.

20 L. Li, S. Fan, C. Zhuang, C. Ban and X. Liu, Customizable Local Superhydrophobic Micro Channels by BNNTs Growth, Microelectron. Eng., 2015, 141, 223-227.

21 T. Hirano, T. Oku and K. Suganuma, Fabrication and Magnetic Properties of Boron Nitride Nanocapsules Encaging Iron Oxide Nanoparticles, Diamond Relat. Mater., 2000, 9, 476-479.

22 L. T. Chadderton and Y. Chen, A Model for the Growth of Bamboo and Skeletal Nanotubes: Catalytic Capillarity, $J$. Cryst. Growth, 2002, 240, 164-169.

23 R. Hoffmann, Solids and Surfaces, 1988.

24 K. Fujimura and A. Miyake, Effect of the Particle Size and Specific Surface Area of Ferric Oxide Catalyst on the Burning Rate of AP/HTPB Solid Propellant, Sci. Technol. Energ. Mater., 2010, 71, 65-69.

25 W. M. Shaheen, Effects of Thermal Treatment and Doping with Cobalt and Manganese Oxides on Surface and 
Catalytic Properties of Ferric Oxide, Mater. Chem. Phys, 2007, 101, 182-190.

26 S. Abbas, A. Abbas, Y. Huang, J. Lin, Z. Liu, Y. Fang and C. Tang, Synthesis of Boron Nitride Nanotubes Using an Oxygen and Carbon Dual-Free Precursor, RSC Adv., 2018, 8, 3989-3995.

27 US Department of Commerce, Nist X-Ray Photoelectron Spectroscopy Database 1, Version 2. NIST NSRDS.

28 J. M. Soler, E. Artacho, J. D. Gale, A. Garcia, J. Junquera, P. Ordejon and D. Sanchez-Portal, The SIESTA Method for Ab Initio Order-N Materials Simulation, Physics, 2001, 14, 2745-2779.

29 E. Artacho, E. Anglada, O. Diéguez, J. D. Gale, A. García, J. Junquera, R. M. Martin, P. Ordejón, J. M. Pruneda and D. Sánchezportal, The SIESTA Method; Developments and Applicability, J. Phys.: Condens. Matter, 2008, 20, 064208.

30 M. Radosavljevic, J. Appenzeller, V. Derycke, R. Martel, P. Avouris, A. Loiseau, J. L. Cochon and D. Pigache, Electrical Properties and Transport in Boron Nitride Nanotubes, Appl. Phys. Lett., 2003, 82, 4131-4133.

31 K. B. Dhungana and R. Pati, Electrical Tuning of Spin Current in a Boron Nitride Nanotube Quantum Dot, Phys. Chem. Chem. Phys., 2014, 16, 7996.

32 C. Kimura, T. Yamamoto and T. Sugino, Study on Electrical Characteristics of Metal/Boron Nitride/Metal and Boron Nitride/Silicon Structures, Diamond Relat. Mater., 2001, 10, 1404-1407.

33 M. Ishigami, J. D. Sau, S. Aloni, M. L. Cohen and A. Zettl, Observation of the Giant Stark Effect in Boron-Nitride Nanotubes, Phys. Rev. Lett., 2005, 94, 056804.
34 K. H. Khoo, M. S. C. Mazzoni and S. G. Louie, Tuning the Electronic Properties of Boron Nitride Nanotubes with Transverse Electric Fields: A Giant Dc Stark Effect, Phys. Rev. B, 2004, 69, 1324-1332.

35 Z. Y. Zhang, C. H. Jin, X. L. Liang, Q. Chen and L. M. Peng, Current-Voltage Characteristics and Parameter Retrieval of Semiconducting Nanowires, Appl. Phys. Lett., 2006, 88, 073102.

36 M. L. Thompson and H. A. Sosa, On the Material Properties and Constitutive Equations of Piezoelectric Poly Vinylidene Fluoride (PVDF), PhD thesis, Drexel University, 2002.

37 Q. M. Wang and L. Eric Cross, Constitutive Equations of Symmetrical Triple Layer Piezoelectric Benders, IEEE Trans. Sonics Ultrason., 1999, 46, 1343-1351.

38 E. J. Mele and P. Král, Electric Polarization of Heteropolar Nanotubes as a Geometric Phase, Phys. Rev. Lett., 2002, 88, 056803.

39 V. Yamakov, C. Park, H. K. Jin, K. E. Wise and C. Fay, Piezoelectric Molecular Dynamics Model for Boron Nitride Nanotubes, Comput. Mater. Sci., 2014, 95, 362-370.

40 B. Gusarov, E. Gusarova, B. Viala, L. Gimeno and O. Cugat, PVDF Piezoelectric Voltage Coefficient In Situ measurements as a Function of Applied Stress, J. Appl. Polym. Sci., 2016, 133, 43248.

41 J. P. Perdew, K. Burke and M. Ernzerhof, Errata: Generalized Gradient Approximation Made Simple, Phys. Rev. Lett., 1996, 77, 3865.

42 M. Ernzerhof and G. E. Scuseria, Assessment of the PerdewBurke-Ernzerhof Exchange-Correlation Functional, J. Chem. Phys., 1999, 110, 5029-5036. 